\title{
Stage IIIA1 Fallopian Tube Cancer AJCC v8
}

National Cancer Institute

\section{Source}

National Cancer Institute. Stage IIIA1 Fallopian Tube Cancer A/CC v8. NCI Thesaurus.

Code C139993.

Stage IIIA1 includes: T1/2, N1, M0. T1: T1: Fallopian tube cancer with tumor limited to fallopian tube(s). T2: Fallopian tube cancer with tumor involving fallopian tubes with pelvic extension below pelvic brim. N1: Positive retroperitoneal lymph nodes only (histologically confirmed). M0: No distant metastasis. (AJCC 8th Ed.) 